\title{
Role of the Basolateral Amygdala in the Storage of Fear Memories across the Adult Lifetime of Rats
}

\author{
Greg D. Gale, ${ }^{1}$ Stephan G. Anagnostaras, ${ }^{2}$ Bill P. Godsil, ${ }^{1}$ Shawn Mitchell, ${ }^{1}$ Takashi Nozawa, ${ }^{1}$ Jennifer R. Sage, ${ }^{1}$ \\ Brian Wiltgen, ${ }^{1}$ and Michael S. Fanselow ${ }^{1}$ \\ Departments of ${ }^{1}$ Psychology and ${ }^{2}$ Neurobiology, University of California, Los Angeles, Los Angeles, California 90095-1563
}

\begin{abstract}
The basolateral amygdala (BLA) is intimately involved in the development of conditional fear. Converging lines of evidence support a role for this region in the storage of fear memory but do not rule out a time-limited role in the memory consolidation. To examine this issue, we assessed the stability of BLA contribution to fear memories acquired across the adult lifetime of rats. Fear conditioning consisted of 10 tone-shock pairings in one context (remote memory), followed 16 months later by 10 additional tone-shock pairings with a novel tone in a novel context (recent memory). Twenty-four hours after recent training, rats were given NMDA or sham lesions of the BLA. Contextual and tone freezing were independently assessed in individual test sessions. Sham-lesioned rats showed high and comparable levels of freezing across all context and tone tests. In contrast, BLA-lesioned rats displayed robust freezing deficits across both recent and remote tests. Subsequent open-field testing revealed no effects of BLA lesions on activity patterns in a dark open field or during bright light exposure. Lesioned rats were able to reacquire normal levels of context-specific freezing after an overtraining procedure ( 76 unsignaled shocks). Together, these findings indicate that BLA lesions do not disrupt freezing behavior by producing hyperactivity, an inability to suppress behavior, or an inability to freeze. Rather, the consistent pattern of freezing deficits at both training-to-lesion intervals supports a role for the BLA in the permanent storage of fear memory.
\end{abstract}

Key words: behavior; learning; memory; NMDA; retrograde; shock

\section{Introduction}

The amygdala has long been implicated in the production of behaviors associated with fear. Extensive work using pavlovian fear conditioning procedures has revealed a role for the basolateral amygdala (BLA) in both the acquisition and expression of conditional fear. Although there remains little doubt that the BLA plays a critical role in fear acquisition, the nature of this involvement remains a matter of debate. A large body of evidence suggests that the BLA provides a substrate for fear memory storage. Lesions, whether made before or shortly after conditioning, produce profound deficits in fear to auditory, visual, and contextual stimuli (Phillips and Ledoux, 1992; Kim and Davis, 1993). Both functional inactivation and receptor antagonism in the BLA before conditioning result in acquisition deficits, although the same manipulations made immediately after conditioning have no effect (Campeau et al., 1992; Fanselow and Kim, 1994; Helmstetter and Bellgowan, 1994; Maren et al., 1996b; Muller et al., 1997; Wilensky et al., 1999). Studies focusing on molecular mechanisms have demonstrated a role for protein synthesis and a number of kinase signaling cascades in the formation of long-

\footnotetext{
Received Sept. 4, 2003; revised Jan. 7, 2004; accepted Jan. 8, 2004.

This work was supported by National Science Foundation Grant 0091487 (M.S.F.).

Correspondence should be sent to Greg Gale, Department of Psychology, University of California, 405 Hilgard Avenue, Los Angeles, CA 90024-1563. E-mail: gale@psych.ucla.edu.

S. G. Anagnostaras's and J. R. Sage's present address: Department of Psychology, University of California, San Diego, La Jolla, CA 92093.

DOI:10.1523/JNEUROSCI.4100-03.2004

Copyright $\odot 2004$ Society for Neuroscience $\quad$ 0270-6474/04/243810-06\$15.00/0
}

term fear memory (Mayford et al., 1996; Bailey et al., 1999; Goosens et al., 2000; Schafe and LeDoux, 2000; Schafe et al., 2000). Importantly, disruption of these processes appears to affect fear memory only during relatively short time windows after training. These findings suggest that processes intrinsic to the BLA are critically involved in supporting associative fear memory.

Although much evidence is consistent with such a memory storage account of BLA function, the existing literature does not universally support this position. On the basis of data from inhibitory avoidance studies, it has been argued that deficits in acquisition after BLA manipulations reflect a role for this region in the consolidation of fear memory. Support for such a consolidation view comes from studies using pharmacological manipulations of the BLA at various times after training. Several agents have been shown to produce enhancements in subsequent avoidance performance when administered to the BLA after training (Liang et al., 1986; Introini-Collison et al., 1996; McGaugh et al., 1988). Importantly, these effects are time limited, showing decreased efficacy with time after training, presumably reflecting the time course of memory consolidation in regions outside the BLA. Additional support for a consolidation view comes from a recent study apparently demonstrating normal avoidance behavior in BLA-lesioned rats that displayed near complete deficits in conditional freezing (Vazdarjanova and McGaugh, 1999). From these data, it has been argued that deficits after BLA lesions do not reflect a failure of memory but rather a disruption of BLAdependent memory consolidation.

A consolidation view would predict that BLA contribution to 
A

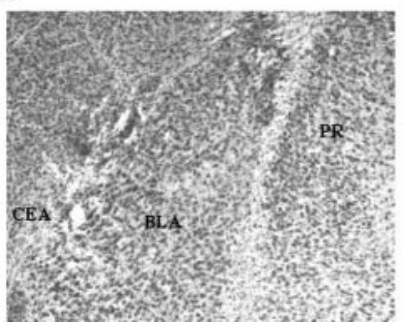

B

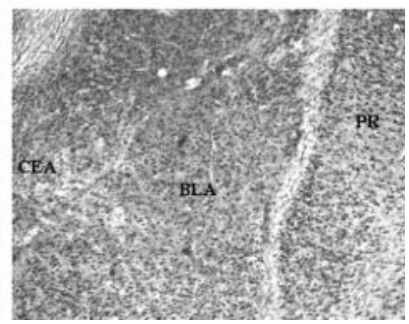

Figure 1. Representative photomicrograph of BLA from sham $(A)$ and lesioned $(B)$ rats. Substantial cell loss in lesioned rats was apparent throughout the BLA. CEA, Central nucleus of the amygdala; PR, perirhinal cortex.

freezing behavior should decrease as time after training increases. Recent studies that have directly addressed this issue do not support this position. With lesions made up to $30 \mathrm{~d}$ after training, no evidence of a temporal gradient has been observed for conditional freezing (Maren et al., 1996a) or fear potentiated startle (Kim and Davis, 1993; Lee et al., 1996). Still, it remains possible that the consolidation period for fear conditioning exceeds the time frame considered in these studies. Indeed, prolonged consolidation gradients, extending well beyond $30 \mathrm{~d}$, have been observed for hippocampal-dependent tasks, including contextual fear conditioning (Zola-Morgan and Squire, 1990; Maren et al., 1997; Anagnostaras et al., 1999). The current study addressed this issue by exposing rats to two fear conditioning episodes separated by 16 months. The contribution of the BLA to these distinct fear memories was then assessed. Additional tests were conducted to address performance interpretations of BLA lesion-induced freezing deficits.

\section{Materials and Methods}

Subjects. Subjects were male Long-Evans rats bred at the University of California at Los Angeles. Rats were housed in individual metal cages and maintained on a 14/10 hr light/dark cycle with access to food and water ad libitum throughout the experiment. Rats were initially handled once daily for 3 consecutive days before training.

Training. All of the animals received tone-shock pairings in one context (remote memory), followed by identical training with a novel tone in a novel context (recent memory) $480 \mathrm{~d}$ later. Figure 1 depicts a sample time line of the experimental procedures. The exact contexts and tones used show little generalization between each other and were counterbalanced. For each conditioning session, the rats were placed into the conditioning chambers, and, after a 2 min baseline period, the animals received 10 tone $(10 \mathrm{sec}, 2$ or $8 \mathrm{kHz}, 90 \mathrm{~dB}$, A scale)-shock (2 sec, $1 \mathrm{~mA}$ ) pairings, with a $64 \mathrm{sec}$ intertrial interval (ITI). Two minutes after the final shock, rats were removed and returned to their home cages.

Surgery. One day after recent training ( $480 \mathrm{~d}$ after remote training), all of the animals were given atropine methyl nitrate $(0.04 \mathrm{mg} / \mathrm{kg}$, i.p. $)$, anesthetized with sodium pentobarbital (50 mg/kg, i.p.), and mounted into a stereotaxic apparatus (David Kopf Instruments, Tujunga, CA). The scalp was incised and retracted, and head position was adjusted to place bregma and lambda in the same horizontal plane. Small burr holes were drilled in the skull above the BLA to accommodate a 28 gauge infusion cannula. A $10 \mu \mathrm{l}$ Hamilton syringe mounted to a infusion pump (Harvard Apparatus, South Natick, MA) and connected to the cannula with polyethylene tubing was used to deliver infusions. For BLA lesions $(n=11)$, NMDA (20 $\mu \mathrm{g} / \mu$ l; Sigma, St. Louis, MO) in $100 \mathrm{~mm} \mathrm{PBS,} \mathrm{pH}$ 7.4 , was infused at a rate of $0.1 \mu \mathrm{l} / \mathrm{min}$ at two sites bilaterally $(3.3 \mathrm{~mm}$ posterior to bregma, $\pm 5.0 \mathrm{~mm}$ lateral to bregma, $8.0 \mathrm{~mm}$ ventral to the skull surface, total volume $0.2 \mu \mathrm{l} ; 2.3 \mathrm{~mm}$ posterior to bregma, $\pm 5.0 \mathrm{~mm}$ lateral to bregma, $7.5 \mathrm{~mm}$ ventral to the skull surface, total volume 0.1 $\mu l)$. Sham rats $(n=11)$ underwent the identical procedure, except that no infusions were made. Representative BLA and sham lesions are shown in Figure 1.

\section{Sample behavioral procedure}

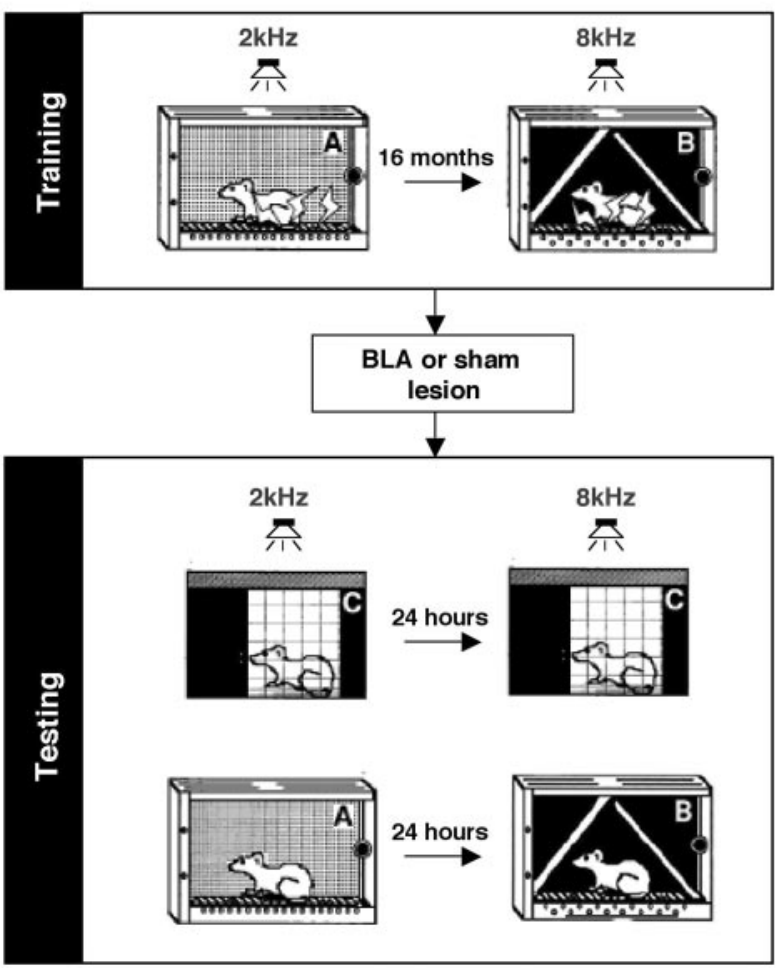

Figure 2. Schematic diagram of sample behavioral procedure. Rats were given pavlovian fear conditioning in one context (remote training), followed 16 months later by additional training in a novel context (recent training). Lesions of the BLA were performed $1 \mathrm{~d}$ after recent training. After recovery, rats were given independent test sessions to assess remote and recent, context and tone memory. The exact contexts and test orders used were counterbalanced

Contexts. The context A environment consisted of aluminum (side walls) and Plexiglas (front, back, and top) chambers $(28 \times 21 \times 22 \mathrm{~cm}$; Lafayette Instruments, Lafayette, IN). The floor of each chamber had 18 stainless steel rods ( $4 \mathrm{~mm}$ diameter, $1.5 \mathrm{~cm}$ apart) connected to a shock scrambler and generator (which, along with internal ventilation fans, supplied background noise of $70 \mathrm{~dB}$, A scale). The chambers were cleaned and scented with a $5 \%$ ammonium hydroxide solution. These computercontrolled (Med-Associates, Lafayette, IN) chambers were in a well lit room separate from the observers. Tones were presented from a speaker in the wall of each chamber. The context B environment was in a separate room. These chambers (same size as above) had a white rear wall inserted and two white plastic side walls $(24 \times 21 \mathrm{~cm})$ placed at $60^{\circ}$ to the floor, forming a triangular enclosure. The floors consisted of 17 staggered rods (two rows, $1 \mathrm{~cm}$ vertically apart; in each row, each rod was $2.6 \mathrm{~cm}$ apart). Background noise $(70 \mathrm{~dB})$ was supplied by a white-noise generator, and the chambers were cleaned and scented with $1 \%$ acetic acid solution. This room was illuminated by a $30 \mathrm{~W}$ red light bulb. The exact contexts and tones used for remote and recent training were counterbalanced (i.e., an equal number of animals were trained in A with $2 \mathrm{kHz}$, followed by $\mathrm{B}$ with $8 \mathrm{kHz} 50$ d later; A8-B2, B2-A8, and B8-A2). Finally, a third context $(\mathrm{C})$ was used for off-baseline tone testing. This context consisted of a stainless steel rack of hanging wire mesh cages $(20 \times 25 \times 18 \mathrm{~cm}$; transparent in the front; opaque side and rear walls). These cages hung over deep, pine wood shavings (providing the background odor), and the entire rack was placed into the same room as the context A chambers. A floor standing fan provided background noise $(55 \mathrm{~dB})$, and the room was illuminated by a $30 \mathrm{~W}$ red light bulb. Tones were delivered from conditioning chambers located behind the rack. Chambers were cleaned with a $5 \%$ sodium hydroxide solution. The transport carriers used to carry the animals were the same as those used for either the A or B context (counterbalanced). 
Freezing. Freezing behavior was defined as the absence of any visible movement (including the vibrissae), except that required for respiration. It was scored according to a blind instantaneous $8 \mathrm{sec}$ time sampling procedure in which each animal was observed eight times per $64 \mathrm{sec}$ interval. For each minute, the number of observations scored as freezing were summed and converted to a percentage (number of freezing observations/total number of observations $\times 100)$. Previous study has revealed that this measure is highly amenable to parametric analysis (Fanselow and Bolles, 1979).

Tests for conditioning. After surgery $(14-15 \mathrm{~d})$, the animals received both a remote and a recent 8 min contextual extinction test on two separate days (order counterbalanced). This was followed $1-2 \mathrm{~d}$ later by remote and recent (counterbalanced) tone tests. For each test, the rats were brought to a novel context (context $\mathrm{C}$, above) for an $8 \mathrm{~min}$ tone extinction test. The animals were placed in the wire cages, and, after a 2 min baseline, either the remote or recent conditioning tone was presented for $6 \mathrm{~min}$. For all tests, freezing was scored continuously. A sample behavioral procedure is depicted in Figure 2 .

Open-field testing. One week after the completion of conditioning tests, general activity and activity suppression were assessed in an open field. The open-field arena was a white translucent polyethylene box (model CB-80; Iris, Pleasant Prairie, WI) with internal dimensions of $69 \times 34 \times 30 \mathrm{~cm}$. Black electrical tape attached to the underside of the floor marked eight squares $(17 \times 17 \mathrm{~cm})$ that were visible from above. The arena was situated on a table in the center of a room that had been decorated with distal cues (posters in the rat's line of sight). Two lamps were situated outside the arena on opposite sides. Each lamp faced the arena and contained a single $100 \mathrm{~W}$ white light bulb. A white-noise generator provided background noise ( $65 \mathrm{~dB}$, A scale). A $25 \mathrm{~W}$ red light bulb that was suspended from the ceiling illuminated the room. For testing, each rat was placed in the arena, and line crossings (defined as the front and rear paws crossing one of the black lines) were recorded for 4 min. The two $100 \mathrm{~W}$ bulbs were then turned on, and line crossings were again recorded for $4 \mathrm{~min}$. Each rat was then removed, and the open-field arena was cleaned with $25 \%$ ethanol in water before the next test session.

Overtraining. Two weeks after open-field testing, lesioned rats were retrained in an overtraining procedure (Maren, 1999). All BLA-lesioned rats were returned to context A. Lighting, olfactory cues, and background noise remained unchanged from previous training in this context. After a 2 minute exposure to the chamber, rats were given 76 unsignaled shocks $(1 \mathrm{~mA}, 2 \mathrm{sec})$ with a $64 \mathrm{sec}$ ITI. Two minutes after the final shock, rats were removed from the chamber and returned to the home cage. On 2 consecutive days after overtraining, BLA-lesioned rats received an $8 \mathrm{~min}$ extinction test in either the overtraining context or a modified $C$ context. For these tests, the $\mathrm{C}$ context was illuminated by a single $100 \mathrm{~W}$ bulb, and background noise was provided by the shock scramblers only $(50 \mathrm{~dB})$ and contained no discernable odors. Chambers were cleaned with a $1 \%$ sodium hydroxide solution between tests. The order in which rats were tested in these two contexts was counterbalanced.

Data analysis. For each memory test, percentage freezing was calculated on a minute-by-minute basis and analyzed by a general multivariate ANOVA. Ambulatory crossovers during the open-field test were calculated for each test minute and similarly analyzed.

\section{Results \\ Context fear}

Two weeks after surgery, fear memory for both the remote and recent contexts was assessed. Each test consisted of an $8 \mathrm{~min}$ extinction test, during which no tones or shocks were presented. Test order for the remote and recent contexts was counterbalanced. Figure $3 A$ depicts the time course of freezing in the remotely conditioned context trained $480 \mathrm{~d}$ before the lesion. BLA lesions produced significant deficits in freezing $\left(F_{(1,20)}=15.90\right.$; $p<0.001)$. The lesion $\times$ minute interaction was also significant $\left(F_{(7,140)}=2.45 ; p<0.03\right)$. Sham-lesioned rats showed an initial increase and then a decrease in freezing, a pattern not observed in the lesioned rats. Figure $3 B$ depicts the time course of freezing in the recently conditioned context trained $1 \mathrm{~d}$ before the lesion.
A

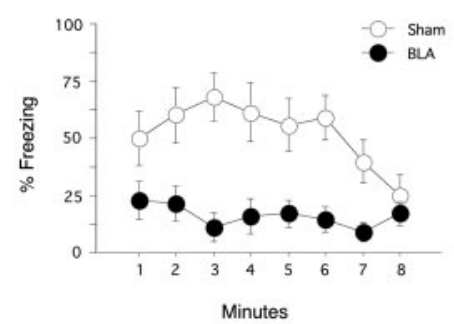

B

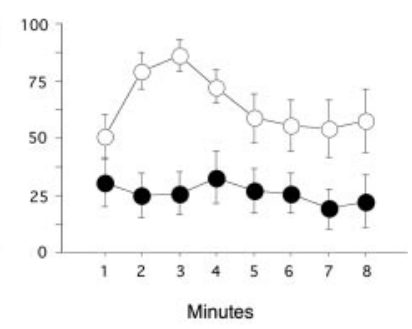

Figure 3. Remote and recent contextual fear. Each test consisted of an 8 min context exposure. No tones or shocks were delivered during these sessions. $A$, Remote contextual fear. When exposed to the remotely trained context, sham rats exhibited high levels of freezing across the 8 min extinction test. In contrast, BLA lesions produced near complete deficits in freezing. $B$, Recent contextual fear. Sham rats exhibited levels of freezing comparable with those observed in the remote context. Again, BLA-lesioned rats displayed significant deficits in freezing across the extinction test.
A

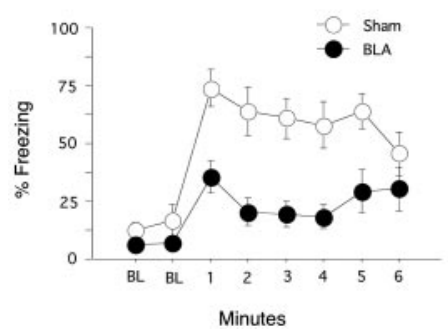

B

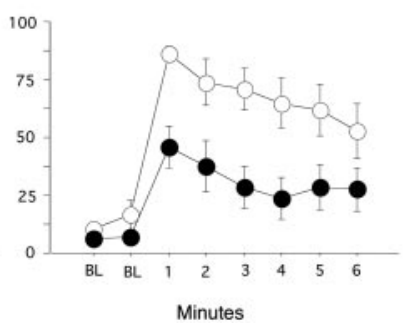

Figure 4. Remote and recent tone fear. Tone tests were conducted by placing the rats in a novel context for a 2 min baseline period (BL), followed by a 6 min continuous tone presentation. $A$, Remote tone fear. Both sham- and BLA-lesioned rats displayed low levels of freezing during the 2 min baseline period. Whereas sham rats displayed high levels of fear during the tone presentation, BLA rats showed pronounced deficits across the test. $B$, Recent tone fear. Baseline freezing in sham- and BLA-lesioned rats was negligible. Sham rats displayed high levels of freezing across the tone test, and BLA rats again showed significant freezing deficits.

Similar to the effects observed on remotely conditioned context fear, BLA lesions severely impaired recently conditioned context fear $\left(F_{(1,20)}=12.48 ; p<0.01\right)$. The lesion $\times$ minute interaction was not significant $\left(F_{(7,140)}=1.74 ; p>0.1\right)$. A comparison of sham-lesioned rats revealed no differences in freezing between recent and remote contexts tests $\left(F_{(1,10)}=0.853 ; p>0.37\right)$. This pattern of results demonstrates that contextual fear depends critically on the BLA regardless of the age of the memory and that no forgetting occurs under these training-to-test intervals.

\section{Tone fear}

After the context tests, memory for remote and recent tone fear was assessed. Each test consisted of a 2 min baseline period, followed by a single 6 min tone presentation of either the tone used during remote or recent training. Test order was counterbalanced. Figure $4 A$ depicts the time course of freezing to the remotely conditioned tone. Negligible levels of freezing were observed during the pre-tone period, and no group differences were observed. In contrast, BLA lesions produced significant freezing deficits to the remote tone $\left(F_{(1,20)}=12.59 ; p<0.01\right)$. The lesion $\times$ minute interaction was also significant $\left(F_{(5,100)}=2.47\right.$; $p<0.04)$, indicating that sham rats showed a general decrease in freezing across the conditioned stimulus presentation not apparent in BLA-lesioned rats. Figure $4 B$ depicts the time course of freezing to the recently conditioned tone. Again, negligible levels of freezing were observed during the pre-tone period, and BLA- 


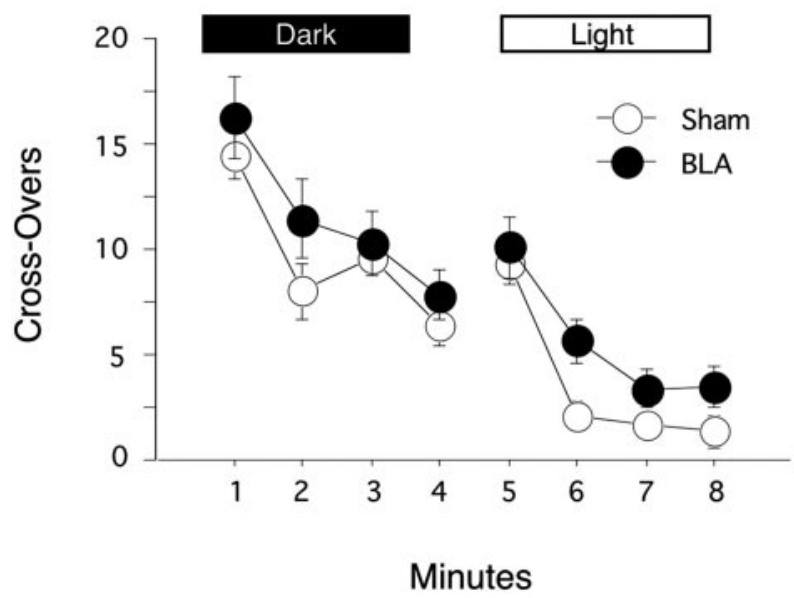

Figure 5. General activity assessment. Rats were placed in a novel open field dimly illuminated by a $25 \mathrm{~W}$ red bulb. Crossovers were scored for $4 \mathrm{~min}$. Two bright lights ( $100 \mathrm{~W}$ ) illuminated the open field for the final 4 min of the test. Sham- and BLA-lesioned rats showed comparable levels of activity and rates of habituation throughout both phases of the test.

lesioned rats did not differ from controls. In contrast, BLA lesions produced significant freezing deficits during presentation of the recent tone $\left(F_{(1,20)}=9.12 ; p<0.01\right)$. The lesion $\times$ minute interaction was not significant $\left(F_{(5,100)}=0.88 ; p>0.4\right)$. Sham rats showed comparable levels of freezing to both recent and remote tones $\left(F_{(1,10)}=0.786 ; p>0.39\right)$. Consistent with the findings from the context tests, the BLA appears to play a critical and stable role in supporting tone fear.

\section{Open-field activity}

Potential changes in activity are of particular concern in investigations of freezing because of the possibility for response competition between activity and freezing. To assess potential activity changes in our BLA-lesioned rats, we examined general activity levels and activity suppression in an open field. Figure 5 depicts the average line crossings during each minute of the 8 min openfield test. The main effect of minute was significant $\left(F_{(7,140)}=\right.$ $44.378 ; p<0.0001$ ), reflecting a general decrease in activity across the session. However, BLA lesions produced no significant effects on activity $\left(F_{(1,20)}=3.07 ; p>0.09\right)$, and no lesion $\times$ time interaction was observed $\left(F_{(7,140)}=0.65 ; p>0.5\right)$. Together, these findings suggest that BLA lesions do not affect general activity levels, habituation, or activity suppression in response to bright light exposure. As such, the freezing deficits observed during the context and tone tests were likely not related to any of these performance factors.

\section{Overtraining}

Figure 6 depicts the average freezing in BLA-lesioned rats during 8 min extinction tests in the overtrained context and a novel context. Freezing levels in the overtrained context were significantly elevated relative to the novel context $\left(t_{(12)}=4.46 ; p<\right.$ $0.001)$. The reliable discrimination between trained and untrained contexts suggests that BLA-lesioned rats are capable of acquiring stimulus-specific conditional fear. Additionally, the freezing levels obtained after the overtraining procedure were comparable with those shown by sham-lesioned rats after the original training protocol. This pattern of data confirms the ability of BLA-lesioned rats to acquire and express contextual freezing that is qualitatively and quantitatively comparable with that of the intact rat.

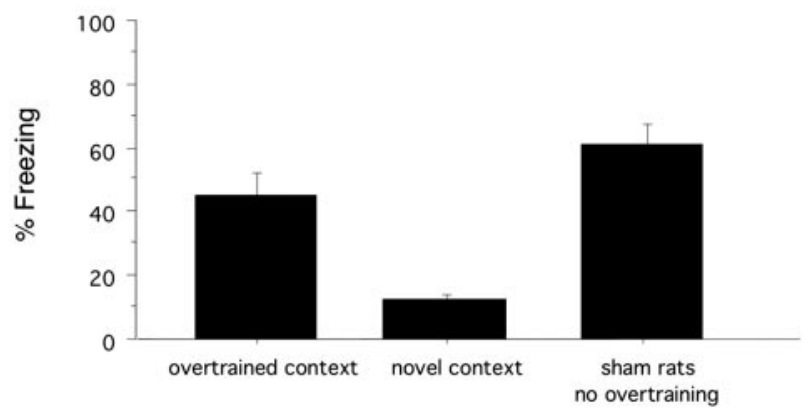

Figure 6. Overtraining. BLA-lesioned rats were returned to one of the two original training contexts and given an overtraining procedure consisting of 76 unsignaled shocks. Over the next $2 \mathrm{~d}$, rats were given 8 min extinction tests in the overtrained context and a novel context. Test order was counterbalanced. Data represents the mean of each 8 min extinction tests. Freezing data for sham rats tested in the same context is provided for comparison.

\section{Discussion}

Converging lines of evidence support a permanent mnemonic role for the BLA in pavlovian fear conditioning. The present study assessed the generality of this position by examining the role of the BLA in the expression of fear memories varying in age from $1 \mathrm{~d}$ to 16 months. Consistent with previous reports, posttraining BLA lesions produced robust freezing deficits to both contextual and discrete auditory stimuli. The critical finding of the present study was that these effects were independent of the training-to-lesion interval. Lesioned rats showed robust and comparable deficits during all recent $(1 \mathrm{~d}$ old $)$ and remote $(16$ month old) memory tests. Because this set of training-to-lesion intervals spans the adult lifetime of rats, the failure to observe any evidence of preserved memory for remote fear memory argues strongly against the existence of prolonged consolidation processes governing the storage of memory supporting conditional freezing. The uniform pattern of freezing deficits after BLA lesions across tests is consistent with a role for the BLA as a substrate supporting memory storage for both auditory and contextual fear.

Given the lack of any temporal gradient for either context or tone fear after post-training BLA lesions, the present findings are readily interpretable as a disruption of fear memory. However, in addition to consolidation accounts of BLA-induced freezing deficits, it has been suggested that deficits after BLA lesions may stem from nonspecific effects on freezing performance (Cahill et al., 1999). By this view, lesions of the BLA produce either general hyperactivity, which competes with the production of the freezing response, or an inability to perform the freezing response. The present study directly addressed both of these possibilities. Assessment of open-field activity levels did not support a hyperactivity interpretation of the observed freezing deficits. Throughout the dark phase, BLA-lesioned rats displayed no evidence of elevated activity relative to sham rats. Rather, both groups showed identical levels of activity and rates of activity habituation. Additionally, data from the light phase provided evidence that BLA lesions have no general effects on the rats' ability to suppress behavior. Exposure to bright light produced a comparable level and rate of activity suppression in both BLA and sham rats. The ability of BLA-lesioned rats to perform the freezing response was assessed through an overtraining procedure. Such training had been shown previously to support reacquisition of contextual freezing after BLA lesions (Maren, 1999). Consistent with the findings of that study, BLA-lesioned rats in the present experiment reacquired levels of contextual freezing comparable 
with those obtained after initial training with an intact BLA. This freezing was specific to the training context because these same rats showed no evidence of fear to an untrained context. These findings show that BLA-lesioned rats are indeed capable of performing the freezing response. Together with previous data demonstrating an intact freezing response to unconditional fear stimuli in rats with BLA lesions (Wallace and Rosen, 2001), these findings do not support performance interpretations of BLAinduced freezing deficits. Importantly, because the activity and overtraining assessments were conducted in the same animals used in the fear conditioning experiments, it is difficult to attribute the observed contextual and tone freezing deficits to performance factors. The reacquisition of context-specific freezing is somewhat surprising given the proposed role of the BLA in fear memory storage. These data suggest that, under extreme training parameters, contextual conditioning can be acquired and expressed independent of the BLA. Rather than undermining the importance of the BLA as a site of memory storage, this finding suggests that other regions can, under a limited set of circumstances, compensate for BLA damage. The circuitry mediating this effect is currently unknown but may involve the periacqueductal gray, a region that plays a critical role in freezing performance (Kim et al., 1993; De Oca et al., 1998).

The within-subjects design used in the present study is assumed to generate fear memories specific to the distinct contexts and tones used during training. The nearly uniform levels of freezing observed across tests could be taken as evidence that control rats acquired recent and remote memories of equal strength. Alternatively, this pattern of results could suggest that sham rats do not readily discriminate between the contexts or tones. In this case, conditional freezing would reflect some common nonspecific memory, and, as such, the present design would not necessarily provide a test of the role of the BLA in consolidation of fear memory. Although the present design does not entirely rule out this possibility, several aspects of the results support the specificity of fear memory. The low levels of freezing observed during the baseline period before the tone tests in context $\mathrm{C}$ together with the high levels of freezing observed during the corresponding periods of the recent and remote contexts tests provide evidence of contextual discrimination. However, this pattern of results demonstrates that sham rats can discriminate between shocked and nonshocked contexts but does not necessarily show that they discriminate between the two training contexts. Data from a previous study does, however, provide direct evidence that these training procedures support independent memories for recent and remote contextual fear. In an identical within-subjects design, using the same apparatus, rats with lesions of the dorsal hippocampus displayed pronounced deficits for recent contextual fear but maintained high levels of remote contextual fear (Anagnostaras et al., 1999). This pattern of data would not be expected if rats had established a common contextual representation or were strongly generalizing between the two contexts. Together, these findings demonstrate specificity of contextual fear memory and validate the behavioral procedure as a tool for assessing the role of the BLA in mediating multiple contextual fear memories. The current results provide no direct evidence for discrimination between the training tones. Assuming that these rats failed to discriminate the recent tone from the remote tone at the time of recent training, it could be argued the recent training simply represents additional conditioning of the remote tone. Given the evidence for contextual discrimination, a lack of tone discrimination would depend on a selective flattening of generalization gradients for tones and not contextual stimuli across the interval between training sessions, a possibility that has not been directly examined. Additionally, the comparable levels of recent and remote tone fear might be expected if rats were discriminating the tones. If rats were treating the recent tone as the remote tone, then recent training should produce a sharp generalization gradient around the recent tone. Subsequent tone testing should then reveal a generalization decrement to the remote tone, a result not supported by the data.

Although the current results join a body of evidence supporting a memory storage view of BLA function, evidence from other aversively motivated tasks suggest a more limited role in fear acquisition. With respect to conditional freezing, a strong prediction from a memory storage perspective is that rats given posttraining BLA lesions should show no savings in the face of retraining. Insofar as the BLA serves as the site of memory storage, lesions of this structure should abolish any memory of training that could serve to accelerate relearning. In an exhaustive analysis of freezing behavior, Maren (2001) found no evidence of savings in BLA-lesioned rats. In contrast, savings have been reported for conditional freezing behavior after similar lesions made before training (Cahill et al., 2000). However, interpretation of these findings is complicated by the use of pretraining lesions. Given the demonstrated role for the BLA in fear acquisition, rats in this study are likely not acquiring memory in the same way as rats with an intact BLA. The critical issue with respect to savings is whether memory acquired with an intact BLA can serve to facilitate reacquisition in the absence of this structure. Only a posttraining lesion design, such as that used by Maren (2001), can adequately address this issue. As such, available evidence provides no support for savings after BLA lesions.

Although the current results favor a storage interpretation of BLA function, they do not rule out a contribution to the storage of memory for certain aspects of the fear conditioning situation in other regions or a time-limited role in these processes. Studies in humans have provided somewhat conflicting results with respect to this issue. Patients with amygdala damage tend to show deficits in the facilitation of declarative memory produced by emotional material (Adolphs et al., 1997, 2000), a finding consistent with a role for the BLA in coordinating storage of declarative memory. However, other evidence supports dissociable BLA contributions to declarative and nondeclarative emotional memory. A study of patients with specific amygdala damage revealed normal declarative fear memory acquisition, despite severe deficits in acquisition of nondeclarative fear memory (Bechara et al., 1995). Thus, even in the absence of BLA activity, the content of declarative memory for the fear conditioning episode appears normal. Together, these findings suggest that the BLA plays a specialized role in encoding the emotional aspects of the fear conditioning situation rather than coordinating the consolidation of declarative memory in extra-amygdala regions.

\section{References}

Adolphs R, Cahill L, Schul R, Babinsky R (1997) Impaired declarative memory for emotional material following bilateral amygdala damage in humans. Learn Mem 4:291-300.

Adolphs R, Tranel D, Denburg N (2000) Impaired emotional declarative memory following unilateral amygdala damage. Learn Mem 7:180-186.

Anagnostaras SG, Maren S, Fanselow MS (1999) Temporally graded retrograde amnesia of contextual fear after hippocampal damage in rats: within-subjects examination. J Neurosci 19:1106-1114.

Bailey DJ, Kim JJ, Sun W, Thompson RF, Helmstetter FJ (1999) Acquisition of fear conditioning in rats requires the synthesis of mRNA in the amygdala. Behav Neurosci 113:276-282.

Bechara A, Tranel D, Damasio H, Adolphs R, Rockland C, Damasio AR (1995) Double dissociation of conditioning and declarative knowledge 
relative to the amygdala and hippocampus in humans. Science 269:1115-1118.

Cahill L, Weinberger NM, Roozendaal B, McGaugh JL (1999) Is the amygdala a locus of "conditioned fear"? Some questions and caveats. Neuron 23:227-228

Cahill L, Vazdarjanova A, Setlow B (2000) The basolateral amygdala complex is involved with, but is not necessary for, rapid acquisition of Pavlovian "fear conditioning." Eur J Neurosci 12:3044-3050.

Campeau S, Miserendino MJ, Davis M (1992) Intra-amygdala infusion of the $\mathrm{N}$-methyl-D-aspartate receptor antagonist AP5 blocks acquisition but not expression of fear-potentiated startle to an auditory conditioned stimulus. Behav Neurosci 106:569-574.

De Oca BM, DeCola JP, Maren S, Fanselow MS (1998) Distinct regions of the periaqueductal gray are involved in the acquisition and expression of defensive responses. J Neurosci 18:3426-3432.

Fanselow MS, Bolles RC (1979) Naloxone and shock-elicited freezing in the rat. J Comp Physiol Psychol 93:736-744.

Fanselow MS, Kim JJ (1994) Acquisition of contextual Pavlovian fear conditioning is blocked by application of an NMDA receptor antagonist D,L2-amino-5-phosphonovaleric acid to the basolateral amygdala. Behav Neurosci 108:210-212.

Goosens KA, Holt W, Maren S (2000) A role for amygdaloid PKA and PKC in the acquisition of long-term conditional fear memories in rats. Behav Brain Res 114:145-152.

Helmstetter FJ, Bellgowan PS (1994) Effects of muscimol applied to the basolateral amygdala on acquisition and expression of contextual fear conditioning in rats. Behav Neurosci 108:1005-1009.

Introini-Collison IB, Dalmaz C, McGaugh JL (1996) Amygdala betanoradrenergic influences on memory storage involve cholinergic activation. Neurobiol Learn Mem 65:57-64.

Kim JJ, Rison RA, Fanselow MS (1993) Effects of amygdala, hippocampus, and periaqueductal gray lesions on short- and long-term contextual fear. Behav Neurosci 107:1093-1098.

Kim M, Davis M (1993) Lack of a temporal gradient of retrograde amnesia in rats with amygdala lesions assessed with the fear-potentiated startle paradigm. Behav Neurosci 107:1088-1092.

Lee Y, Walker D, Davis M (1996) Lack of a temporal gradient of retrograde amnesia following NMDA-induced lesions of the basolateral amygdala assessed with the fear-potentiated startle paradigm. Behav Neurosci $110: 836-839$.

Liang KC, Juler RG, McGaugh JL (1986) Modulating effects of posttraining epinephrine on memory: involvement of the amygdala noradrenergic system. Brain Res 368:125-133.

Maren S (1999) Neurotoxic basolateral amygdala lesions impair learning and memory but not performance of conditional fear in rats. J Neurosci 19:8696-8703.
Maren S (2001) Is there savings for pavlovian fear conditioning after neurotoxic basolateral amygdala lesions in rats? Neurobiol Learn Mem 76: $268-283$.

Maren S, Aharonov G, Fanselow MS (1996a) Retrograde abolition of conditional fear after excitotoxic lesions in the basolateral amygdala of rats: absence of temporal gradient. Behav Neurosci 110:718-726.

Maren S, Aharonov G, Stote DL, Fanselow MS (1996b) N-methyl-Daspartate receptors in the basolateral amygdala are required for both acquisition and expression of conditional fear in rats. Behav Neurosci 110:1365-1374.

Maren S, Aharonov G, Fanselow MS (1997) Neurotoxic lesions of the dorsal hippocampus and Pavlovian fear conditioning in rats. Behav Brain Res 88:261-274.

Mayford M, Bach ME, Huang YY, Wang L, Hawkins RD, Kandel ER (1996) Control of memory formation through regulated expression of a CaMKII transgene. Science 274:1678-1683.

McGaugh JL, Introini-Collison IB, Nagahara AH (1988) Memoryenhancing effects of posttraining naloxone: involvement of betanoradrenergic influences in the amygdaloid complex. Brain Res 446:37-49.

Muller J, Corodimas KP, Fridel Z, LeDoux JE (1997) Functional inactivation of the lateral and basal nuclei of the amygdala by muscimol infusion prevents fear conditioning to an explicit conditioned stimulus and to contextual stimuli. Behav Neurosci 111:683-691.

Phillips RG, LeDoux JE (1992) Differential contribution of amygdala and hippocampus to cued and contextual fear conditioning. Behav Neurosci 106:274-285.

Schafe GE, LeDoux JE (2000) Memory consolidation of auditory pavlovian fear conditioning requires protein synthesis and protein kinase $\mathrm{A}$ in the amygdala. J Neurosci 20:RC96(1-5).

Schafe GE, Atkins CM, Swank MW, Bauer EP, Sweatt JD, LeDoux JE (2000) Activation of ERK/MAP kinase in the amygdala is required for memory consolidation of pavlovian fear conditioning. J Neurosci 20:8177-8187.

Vazdarjanova A, McGaugh JL (1999) Basolateral amygdala is involved in modulating consolidation of memory for classical fear conditioning. J Neurosci 19:6615-6622.

Wallace KJ, Rosen JB (2001) Neurotoxic lesions of the lateral nucleus of the amygdala decrease conditioned fear but not unconditioned fear of a predator odor: comparison with electrolytic lesions. J Neurosci 21:3619-3627.

Wilensky AE, Schafe GE, LeDoux JE (1999) Functional inactivation of the amygdala before but not after auditory fear conditioning prevents memory formation. J Neurosci 19:RC48(1-5).

Zola-Morgan SM, Squire LR (1990) The primate hippocampal formation: evidence for a time-limited role in memory storage. Science 250:288-290. 